See discussions, stats, and author profiles for this publication at: https://www.researchgate.net/publication/282305118

\title{
Binary Flower Pollination Algorithm and Its Application to Feature Selection
}

Article in Studies in Computational Intelligence · January 2015

DOI: 10.1007/978-3-319-13826-8_5

CITATIONS

41

4 authors:

Douglas Rodrigues

Universidade Federal de São Carlos

22 PUBLICATIONS 550 CITATIONS

SEE PROFILE

André N. DE Souza

São Paulo State University

99 PUBLICATIONS 698 CITATIONS

SEE PROFILE

Some of the authors of this publication are also working on these related projects:

Project RBM-based works View project

Project Drilling Analysis View project
READS

940

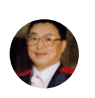

Xin-She Yang

Middlesex University, UK

483 PUBLICATIONS 28,899 CITATIONS

SEE PROFILE

João Paulo Papa

São Paulo State University

311 PUBLICATIONS 3,244 CITATIONS

SEE PROFILE 


\title{
Binary Flower Pollination Algorithm and Its Application to Feature Selection
}

\author{
Douglas Rodrigues, Xin-She Yang, André Nunes de Souza and João Paulo Papa
}

\begin{abstract}
The problem of feature selection has been paramount in the last years, since it can be so important as the classification step itself. The main goal of feature selection is to find out the subset of features that maximizes some fitness function, which can be some classifier's accuracy, or even the computational burden for extracting each feature, for instance. Therefore, such approaches are likely to be modeled as being optimization tasks, as well as evolutionary-based ones. In this chapter, we evaluate a binary-constrained version of the Flower Pollination Algorithm (FPA) for feature selection purposes, in which the optimization search space is carried out on a boolean lattice where each possible solution a string of bits that denote whether a feature will be used to compose the final set. Experiments over some public and private datasets against with Particle Swarm Optimization, Harmony Search and Firefly Algorithm have demonstrated the suitability of FPA for feature selection.
\end{abstract}

\section{Introduction}

Machine learning techniques have been actively pursed in the last years, since there is an increasing number of applications that make use of some sort of intelligencebased decision process. Roughly speaking, a standard workflow for tackling such problems can be divided in four phases: (i) to preprocess the data (signal or im-

Douglas Rodrigues

Department of Computing, UNESP, Bauru/SP, Brazil, e-mail: douglasrodrigues.dr@gmail.com

Xin-She Yang

School of Science and Technology, Middlesex University, London, UK e-mail: x.yang@mdx.ac.uk

André Nunes de Souza

Department of Electrical Engineering, UNESP, Bauru/SP, Brazil, e-mail: andrejau @ feb.unesp.br

João Paulo Papa

Department of Computing, UNESP, Bauru/SP, Brazil, e-mail: papa@ @c.unesp.br 
age filtering, for instance); (ii) to extract features; (ii) to train a machine learning technique, and finally (iv) to evaluate its effectiveness over an unseen (test) data [3].

One of the most important steps concerns the feature extraction, which aims at finding the most important subset of features that leads to the best recognition rates. There are situations we may obtain the same accuracy as before (with the original set of features) even after feature selection, but we can save computational effort by avoiding to extract some features that are too costly for that.

Several studies have modeled the problem of feature selection as an optimization task, since the idea is to find out the subset of features that maximizes the accuracy of a given classifier, or minimizes its error over some validating set, for instance. Such environment is friendly to the application of evolutionary optimization techniques, which have as the main feature to imitate living beings for solving complex tasks. The reader can refer to some interesting literature such as the Binary Particle Swarm Optimization (BPSO) [5], Binary Firefly Algorithm (BFA) [4], Binary Harmony Search (BHS) [14], Binary Gravitational Search Algorithm (BGSA) [16], Binary Cuckoo Search (BCS) [17], Binary Charged System Search (BCSS) [19], and Binary Bat Algorithm (BBA) [18].

Yang and Honavar [23] presented a multicriteria Genetic Algorithm (GA) to the problem of feature selection, in which the idea was to optimize both the accuracy and the feature extraction computational costs. Later on, Oh et al. [10] proposed a hybrid GA to tackle the same problem with a better final performance. Also, there are many papers that address feature selection with Ant Colonization [21, 7, 2], among others. The main idea consists of reducing the number of possible paths visited by ants in some works, as well as modified pheromone update rules. Other approaches such as Artificial Bee Colony [20, 9] and Gravitational Search Algorithm $[15,1]$ have been also employed to the same context.

Basically, the idea of these methods is to convert the position of the agents (bats, particles, harmonies, etc.) into binary-valued coordinates, which are represented by a string of bits, each denoting the presence or absence of a feature. One can consider the problem of feature selection as a search task in a boolean lattice, in which the number of dimensions stands for the number of features. As the original versions of the evolutionary optimization techniques were proposed to handle continuousvalued problems, the idea is to apply a discretization function (usually a constrained sigmoid function) to map the agents to the boolean lattice.

Very recently, Yang $[25,26]$ proposed the Flower Pollination Algorithm (FPA), which is inspired by the flower pollination process of flowering plants. This approach has demonstrated interesting results for traditional (continuous-valued) optimization problems, which motivated us to extend it to solve binary optimization tasks. In this case, we have tackled the problem of feature selection to evaluate the proposed approach, which is called Binary Flower Pollination Algorithm (BFPA). In regard to the fitness function, we have used a classifier's effectiveness over a validating set: as we need to train a classifier every time an agent (pollen) changes its position, we need a fast classifier. For such purpose, we opted to employ the Optimum-Path Forest (OPF) [12, 13], which has demonstrated very promising results in several application, being also parameter-independent. The proposed ap- 
proach has been compared against with BPSO, BFA and BHS in several datasets, being their results analized through statistical tools.

The remainder of this chapter is organized as follows. Section 2 introduces the theory background about FPA and OPF techniques. Sections 3 and 4 present the methodology and the experimental results, respectively. Finally, Section 5 states conclusions and future works.

\section{Theoretical Background}

In this section we briefly review some of the main important concepts regarding the techniques employed in this paper, as well as the proposed Binary Flower Pollination Algorithm.

\subsection{Flower Pollination Algorithm}

The Flower Pollination Algorithm was proposed by Yang [25], being inspired by the pollination process of flowering plants. The FPA is governed by four basic rules:

1. Biotic cross-pollination can be considered as a process of global pollination, and pollen-carrying pollinators move in a way that obeys Lévy flights.

2. For local pollination, abiotic pollination and self-pollination are used.

3. Pollinators such as insects can develop flower constancy, which is equivalent to a reproduction probability that is proportional to the similarity of two flowers involved.

4. The interaction or switching of local pollination and global pollination can be controlled by a switch probability $p \in[0,1]$, slightly biased towards local pollination.

However, it is necessary that the aforementioned basic rules be converted into appropriate updating equations. For example, in the global pollination step, flower pollen gametes are carried by pollinators such as insects, and pollen can travel over a long distance because insects can often fly and move over a much longer range [25]. Therefore, Rules 1 and 3 can be represented mathematically as:

$$
x_{i}^{(t+1)}=x_{i}^{t}+\alpha L(\lambda)\left(g_{*}-x_{i}^{t}\right)
$$

where

$$
L(\lambda)=\frac{\lambda \cdot \Gamma(\lambda) \cdot \sin (\lambda)}{\pi} \cdot \frac{1}{s^{1+\lambda}}, \quad s \gg s_{0}>0
$$

where $x_{i}^{t}$ is the pollen $i$ (solution vector) at iteration $t, g_{*}$ is the current best solution found among all solutions at the current generation, and $\alpha$ is a scaling factor to control the step size, $L(\lambda)$ is the Lévy flights step size, that corresponds to the strength of the pollination, $\Gamma(\lambda)$ stands for the gamma function, and $s$ is the step size. Since 
insects may move over a long disntace with various distance steps, a Lévy flight can be used to mimic this characteristic efficiently.

For local pollination, both Rules 2 and 3 can be represented as:

$$
x_{i}^{(t+1)}=x_{i}^{t}+\varepsilon\left(x_{j}^{t}-x_{k}^{t}\right),
$$

where $x_{j}^{t}$ and $x_{k}^{t}$ stand for the pollen from different flowers $j$ and $k$ of the same plant species, respectively. This mimics flower constancy in a limited neighbourhood. Mathematically, if $x_{j}^{t}$ and $x_{k}^{t}$ come from the same species or are selected from the same population, it equivalently becomes a local random walk if $\varepsilon$ is drawn from a uniform distribution in [0,1]. In order to mimic the local and global flower pollination, a switch probability (Rule 4) or proximity probability $p$ is used.

\subsubsection{Binary Flower Pollination Algorithm}

In the standard FPA, the solutions are updated in the search space towards continuousvalued positions. However, in the proposed Binary Flower Pollination Algorithm the search space is modelled as a $d$-dimensional boolean lattice, in which the solutions are updated across the corners of a hypercube. In addition, as the problem is to select or not a given feature, a solution binary vector is employed, where 1 corresponds whether a feature will be selected to compose the new dataset, and 0 otherwise. In order to build this binary vector, we have employed Equation 5 just after Equation 3, which can restrict the new solutions to only binary values:

$$
\begin{gathered}
S\left(x_{i}^{j}(t)\right)=\frac{1}{1+e^{-x_{i}^{j}(t)},} \\
x_{i}^{j}(t)= \begin{cases}1 & \text { if } S\left(x_{i}^{j}(t)\right)>\sigma, \\
0 & \text { otherwise }\end{cases}
\end{gathered}
$$

where $\sigma \sim U(0,1)$. Algorithm 1 presents the proposed BFPA for feature selection using the recognition rate of the OPF classifier as the objective function. Note the proposed approach can be used with any other supervised classification technique.

Lines $1-4$ initialize each pollen's position as being a binary string with random values, as well as the fitness value $f_{i}$ of each individual $i$. The main loop in Lines $6-27$ is the core of the proposed algorithm, in which the inner loop in Lines 7-13 is responsible for creating the new training $Z_{1}^{\prime}$ and evaluating sets $Z_{2}^{\prime}$, and then OPF is trained over $Z_{1}^{\prime}$ and it is used to classify $Z_{2}^{\prime}$. The recognition accuracy over $Z_{2}^{\prime}$ is stored in $a c c$ and then compared with the fitness value $f_{i}$ (accuracy) of individual $i$ : if the later is worse than $a c c$, the old fitness value is kept; in the opposite case, the fitness value is then updated. Lines $12-13$ update the best local position of the current pollen. Lines $14-18$ update the global optimum, and the last loop (Lines 19 - 27) moves each pollen to a new binary position restricted by Equation 5 (Lines $25-27)$. 


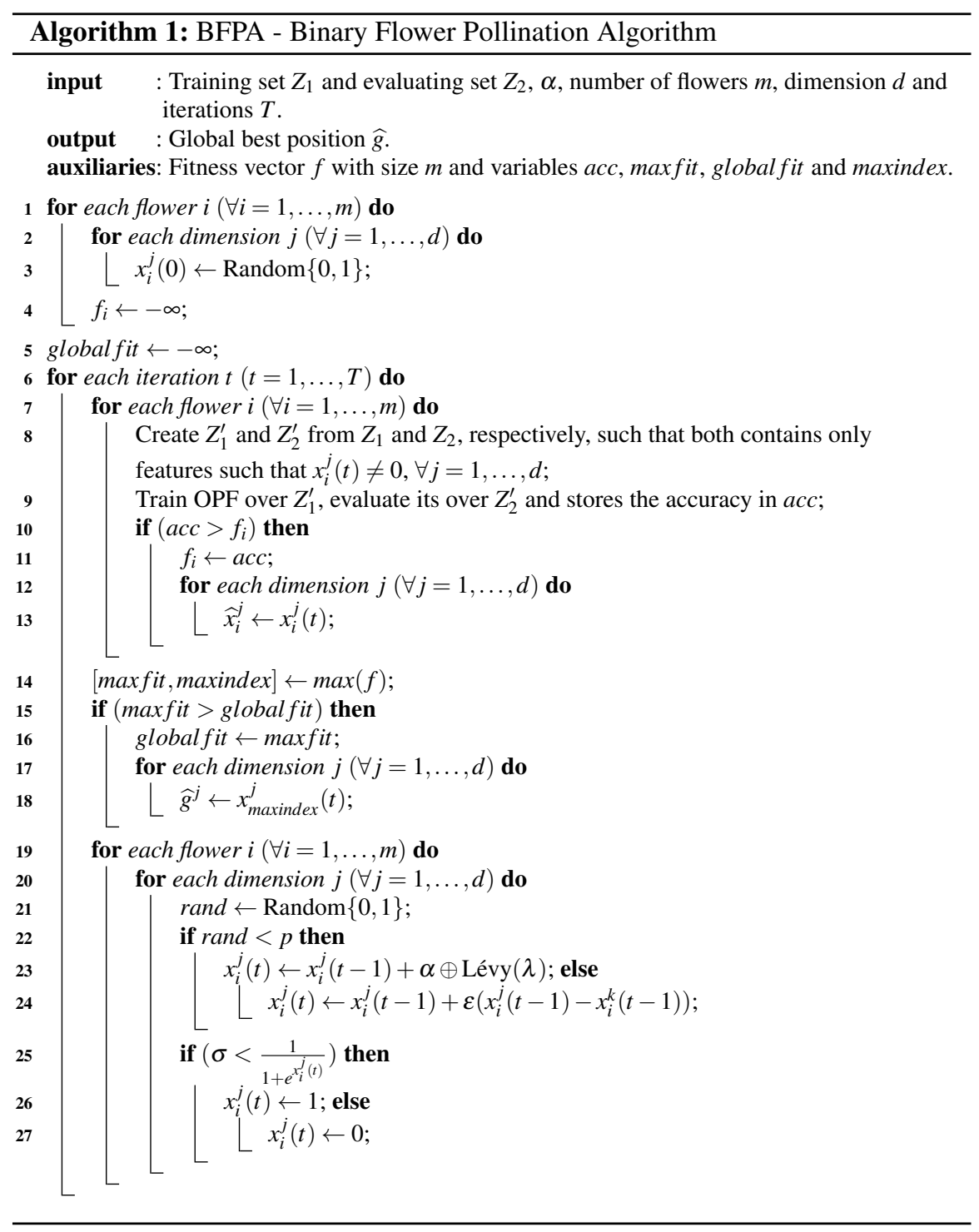

\subsection{Optimum-path Forest classifier}

The Optimum-Path Classifier $[12,13]$ works by modelling the samples as graph nodes, whose arcs are defined by an adjacency relation and weighted by some distance function. Further, a role competition process between some key nodes (prototypes) is carried out in order to partition the graph into optimum-path trees (OPTs) according to some path-cost function. Therefore, to design an Optimum-Path Forest- 
based classifier, one needs to define: (i) an adjacency relation, (ii) a path-cost function and (iii) a methodology to estimate prototypes.

Suppose we have a fully labeled dataset $Z=Z_{1} \cup Z_{2}$, in which $Z_{1}$ and $Z_{2}$ stand for training and test sets, respectively. Let $S \subset Z_{1}$ be a set of prototypes of all classes (i.e., key samples that best represent the classes). Let $\left(Z_{1}, A\right)$ be a complete graph whose nodes are the samples in $Z_{1}$ and any pair of samples defines an arc in $A=$ $Z_{1} \times Z_{1}$. Let $\pi_{s}$ be a path in the graph that ends in sample $s \in Z_{1}$, and $\left\langle\pi_{s} \cdot(s, t)\right\rangle$ the concatenation between $\pi_{s}$ and the $\operatorname{arc}(s, t), t \in Z_{1}$. In this chapter, we employ a path-cost function that returns the maximum arc-weight along a path in order to avoid chains, and also to give the idea of connectivity between samples. This pathcost function is denoted here as $\Psi$, and it can be computed as follows:

$$
\begin{aligned}
\Psi(\langle s\rangle) & = \begin{cases}0, & \text { if } s \in S \\
+\infty, & \text { otherwise },\end{cases} \\
\Psi\left(\pi_{s} \cdot\langle s, t\rangle\right) & =\max \left\{\Psi\left(\pi_{s}\right), d(s, t)\right\},
\end{aligned}
$$

in which $d(s, t)$ means the distance between nodes $s$ and $t$. Thus, the objective of the Optimum-Path Forest algorithm (supervised version) is to minimize $\Psi\left(\pi_{t}\right), \forall t \in Z_{1}$.

An optimal set of prototypes $S^{*}$ can be found by exploiting the theoretical relation between minimum-spanning tree and optimum-path tree for $\Psi$. By computing a minimum-spanning tree in the complete graph $\left(Z_{1}, A\right)$, we obtain a connected acyclic graph whose nodes are all samples of $Z_{1}$ and the arcs are undirected and weighted by the distances $d$ between adjacent samples. The spanning tree is optimum in the sense that the sum of its arc weights is the minimum as compared to any other spanning tree in the complete graph. In the minimum-spanning tree, every pair of samples is connected by a single path, which is optimum according to $\Psi$. Thus, the minimum-spanning tree contains one optimum-path tree for any selected root node. The optimum prototypes are the closest elements of the minimum-spanning tree with different labels in $Z_{1}$.

The Optimum-Path Forest training phase consists, essentially, of starting the competition process between prototypes in order to minimize the cost of each training sample. At the final of such procedure, we obtain an optimum-path forest, which is a collection of optimum-path threes rooted at each prototype. A sample connected to an OPT means that it is more strongly connected to the root of that tree than to any other root in the forest.

Further, in the classification phase, for any sample $t \in Z_{2}$, we consider all arcs connecting $t$ with samples $s \in Z_{1}$, as though $t$ were part of the training graph. Considering all possible paths from $S^{*}$ to $t$, we find the optimum path $P^{*}(t)$ from $S^{*}$ and label $t$ with the class $\lambda(R(t))$ of its most strongly connected prototype $R(t) \in S^{*}$. This path can be identified incrementally, by evaluating the optimum cost $C(t)$ as:

$$
C(t)=\min \{\max \{C(s), d(s, t)\}\}, \forall s \in Z_{1} .
$$


Let the node $s^{*} \in Z_{1}$ be the one that satisfies (Equation 7) (i.e., the predecessor $P(t)$ in the optimum path $\left.P^{*}(t)\right)$. Given that $L\left(s^{*}\right)=\lambda(R(t))$, the classification simply assigns $L\left(s^{*}\right)$ as the class of $t$.

\section{Methodology}

In this section, we present the methodology used to evaluate the performance of BFPA. Details about the dataset used, experimental setup and the compared techniques are also provided.

\subsection{Datasets}

Table 1 presents the datasets used in this work ${ }^{1}$. Such datasets differ on the number of samples, features and also classes. Therefore, the idea is to evaluate the proposed approach in different contexts.

Table 1 Description of the benchmarking datasets.

\begin{tabular}{lccc}
\hline Dataset & \multicolumn{3}{c}{ \# samples \# features } \\
\hline GLI-85 classes \\
SMK-CAN-187 & 185 & 22,283 & 2 \\
TOX-171 & 171 & 5,993 & 2 \\
AR10P & 130 & 2,400 & 4 \\
NTL $_{c}$ & 4,952 & 8 & 2 \\
NTL $_{i}$ & 3,182 & 8 & 2 \\
\hline
\end{tabular}

The two last datasets, i.e., $\mathrm{NTL}_{c}$ and $\mathrm{NTL}_{i}$, are related with non-technical losses detection in comercial and industrial profiles, respectively. These are private datasets obtained by a Brazilian electrical power company. Such sort of problem is of great interest of electrical power companies, mainly in Brazil, in which the amount of losses in energy thefts reaches up to $20 \%$ in some regions. Therefore, the characterization of illegal consumers, i.e., to find out the most important features that allow us to identify them, is so important as to effective recognize them.

\footnotetext{
${ }^{1}$ The first four datasets can be found on http://featureselection.asu.edu/datasets.php
} 


\subsection{Nature-Inspired Metaheuristic Algorithms}

In this work, we have also employed three others evolutionary optimization techniques for comparison purposes. A brief detail about each of them is given below.

Particle Swarm Optimization (PSO): is inspired on the social behavior of bird flocking or fish schooling [8]. The fundamental idea is that each particle represents a potential solution which is updated according to its own experience and from its neighbors' knowledge. The motion of an individual particle for the optimal solution is governed through its position and velocity interactions, and also by its own previous best performance and the best performance of their neighbors.

Firefly Algorithm (FA): it was also proposed by Yang [24], being derived from the flash attractiveness of fireflies for mating partners (communication) and attracting potential preys. The brightness of a firefly at a given position is determined by the value of the objective function in that position. Each firefly is attracted by a brighter firefly through the attraction factor.

Harmony Search (HS): is a meta-heuristic algorithm inspired in the improvisation process of music players [6]. Musicians often improvise the pitches of their instruments searching for a perfect state of harmony. The main idea is to use the same process adopted by musicians to create new songs to obtain a near-optimal solution according to some fitness function. Each possible solution is modelled as a harmony, and each musical note corresponds to one decision variable.

Notice we have used the binary optimization version of each aforementioned technique, i.e., Binary PSO (BPSO) [5], Binary Firefly (BFA) [11, 4], as well as Binary HS (BHS) [14].

\subsection{Experimental Setup}

Firstly, the dataset $Z$ is randomly partitioned in $N$ folds, i.e., $Z=F_{1} \cup F_{2} \cup \cdots \cup F_{N}$. For each fold $F_{i}$, we train a given instance of the OPF classifier over it, for further evaluation of another fold $F_{j}, i \neq j$. Therefore, the classification accuracy over $F_{j}$ is then used as the fitness function to guide the optimization algorithms for selecting the most representative set of features. Each agent of the population (pollen, particle, firefly, harmony) in the meta-heuristic algorithm is associated with a string of bits denoting the presence or absence of a feature. Thus, for each agent, we construct a classifier from the training set $F_{i}$ only with the selected features, say that $F_{i}^{*}$, and assigns the accuracy over $F_{j}$ as the fitness function. As long as the procedure converges, i.e, all generations of a population were computed, the agent with the highest fitness value encodes a solution with the best compacted set of features.

After that, we build a classification model using the training set with the selected features $\left(F_{i}^{*}\right)$, and we also evaluate the quality of the solution through a classification process over the test set, which is built over the remaining folds in $Z \backslash\left\{F_{i} \cup F_{j}\right\}$. This procedure is conducted for each fold $F_{i}$ in the dataset to be part of the training set, and thus we have $N(N-1)$ accuracies in the final of the process, which will be 
averaged for comparison purposes. Figure 1 illustrates the methodology described above.

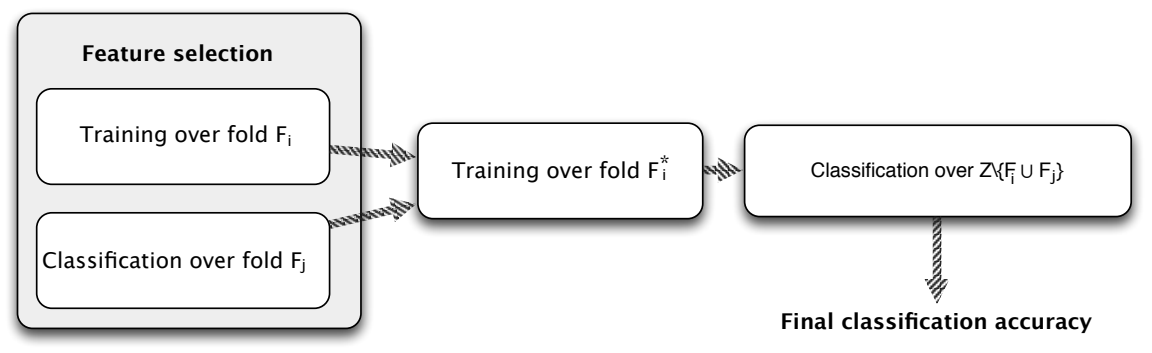

Fig. 1 Proposed methodology to evaluate the compared techniques.

In regard to the recognition rate, we used an accuracy measure proposed by Papa et al. [12]. If there are two classes, for example, with very different sizes and a classifier always assigns the label of the largest class, its accuracy will fall drastically due to the high error rate on the smallest class. The accuracy is measured by taking into account that the classes may have different sizes in a testing set $F_{j}$. Let us define:

$$
e_{i, 1}=\frac{F P_{i}}{\left|F_{j}\right|-\left|F_{j}^{i}\right|}
$$

and

$$
e_{i, 2}=\frac{F N_{i}}{\left|F_{j}^{i}\right|}, i=1,2, \ldots, C,
$$

where $C$ stands for the number of classes, $\left|F_{j}^{i}\right|$ concerns with the number of samples in $F_{j}$ that come from class $i$, and $F P_{i}$ and $F N_{i}$ stand for the false positives and false negatives for class $i$, respectively. That is, $F P_{i}$ is the number of samples from other classes that were classified as being from the class $i$ in $F_{j}$, and $F N_{i}$ is the number of samples from the class $i$ that were incorrectly classified as being from other classes in $F_{j}$. The error terms $e_{i, 1}$ and $e_{i, 2}$ are then used to define the total error from class $i$ :

$$
E_{i}=e_{i, 1}+e_{i, 2} .
$$

Finally, the accuracy Acc is then defined as follows:

$$
A c c=1-\frac{\sum_{i=1}^{C} E_{i}}{2 C} .
$$




\section{Experimental results}

In this section, we discussed the experimental results regarding the proposed approach for feature selection. The results presented in this section stand for the mean accuracy and standard deviation over 25 rounds using the methodology presented in Section 3.3. Since the evolutionary optimization algorithms are non-deterministic, such approaches seem to be robust to avoid biased results. The optimization algorithms (BFPA, BPSO, BHS and BFA) were implemented in C language following the guidelines provided by their references. The experiments were executed in a computer with a Pentium Intel Core $i 5^{\circledR} 3.20 \mathrm{Ghz}$ processor, 4 GB of RAM and Linux Ubuntu Desktop LTS 10.04 as the operational system.

Table 2 presents the parameters used for each optimization technique employed in this work. The $c_{1}$ and $c_{2}$ parameters of PSO control the pace during the particles' movement, and the "Harmony Memory Considering Rate" (HMCR) of BHS stands for the amount of information that will be used from the artist's memory (songs that have been already composed) in order to compose a new harmony. In regard to BFA, $\alpha$ and $\beta_{0}$ are related with the step size of a firefly, and $\gamma$ stands for the light absorption coefficient. In addition, we have used a population of 30 agents and 100 iterations for all techniques, with such values being an empirical set.

\begin{tabular}{|l|c|}
\hline Technique & Parameters \\
\hline \hline BPSO & $c_{1}=c_{2}=2$ \\
BFA & $\gamma=0.8, \beta_{0}=1.0, \alpha=0.01$ \\
BHS & HMCR $=0.9$ \\
BFPA & $\alpha=1.0, p=0.8$ \\
\hline
\end{tabular}

Table 2 Parameters used for each meta-heuristic optimization technique. Notice the inertia weight $w$ for PSO was linearly decreased from 0.9 to 0.4 during the convergence process.

Figure 2 displays the mean accuracy results using the proposed methodology (Section 3.3). One can observe the feature selection techniques can slightly improve the results obtained using the original datasets, i.e., without feature selection. The second point is that all techniques achieved quite similar results. Therefore, the results showed BFPA is suitable for feature selection tasks. We have also performed the statistical Wilcoxon Signed-Rank Test [22] to verify whether there is a significant difference between BFPA and the other techniques used in this work (considering the OPF recognition rate). Table 3 displays the $p$-values, being the bold ones the situations in which BFPA and the respective technique have obtained different performances, i.e., when the $p$-values are lower than a significance level of $\alpha=0.05$.

One can observe there is a statistical difference between BFPA and BPSO, and BFPA and BFA for AR10P dataset, and also a difference between BFPA and BHS considering TOX-171 dataset. Figure 3 displays the convergence rates of all tech- 
niques considering the datasets employed in this work. Such recognition rates are the ones obtained over the validating set $F_{j}$, as depicted in Figure 1.

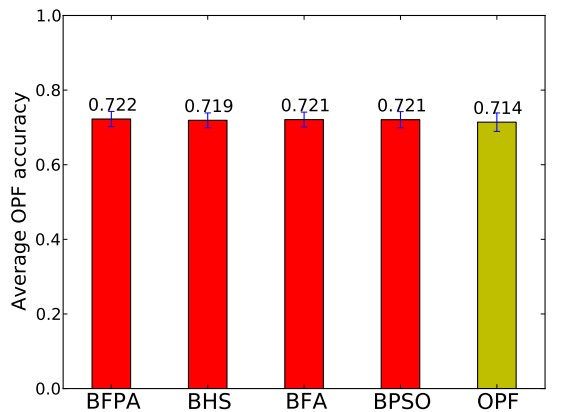

(a)

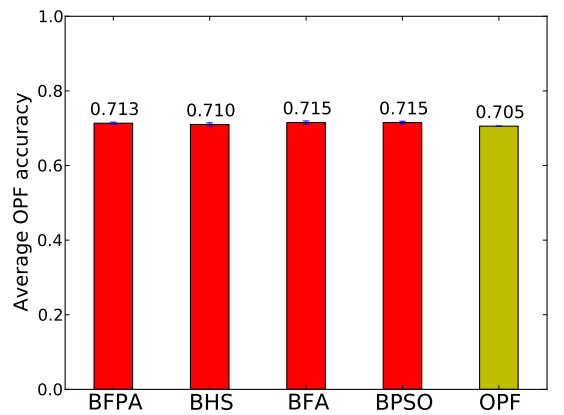

(c)

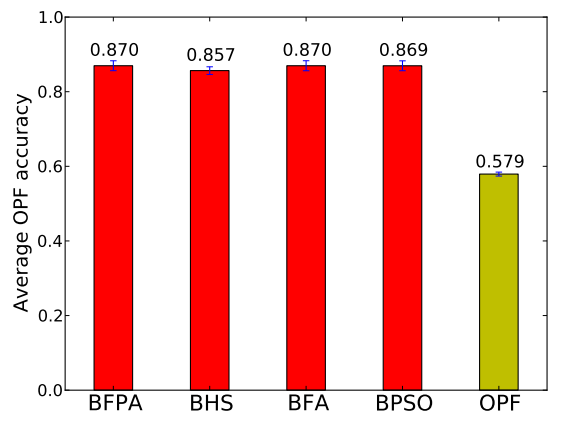

(e)

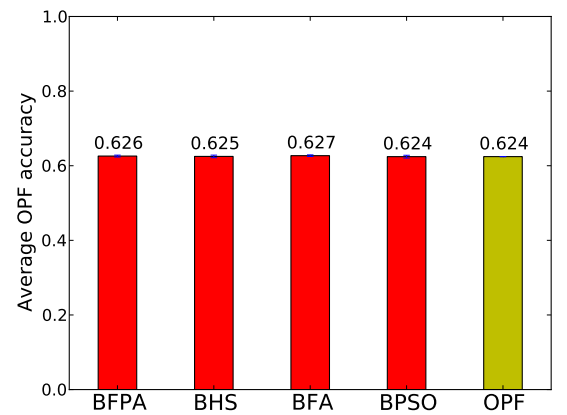

(b)

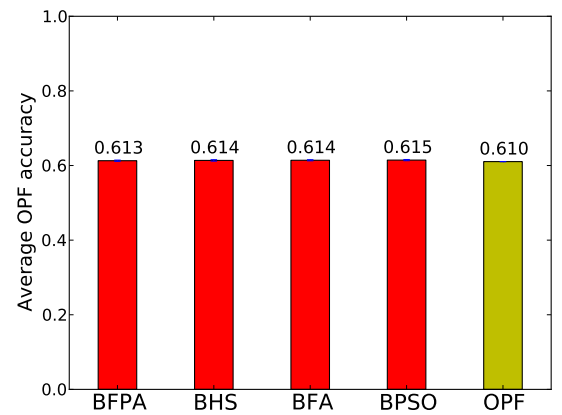

(d)

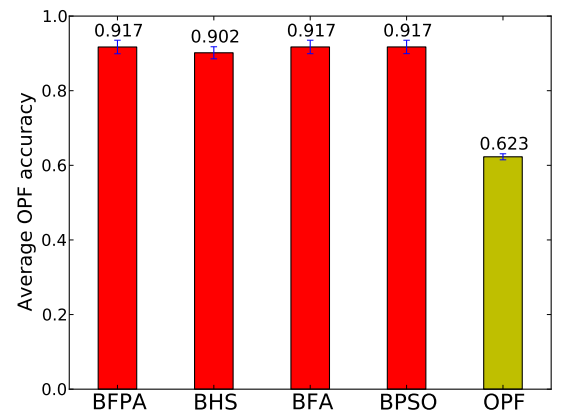

(f)

Fig. 2 Average OPF accuracy over (a) GLI-85, (b) SMK-CAN-187, (c) TOX-171, (d) AR10P, (e) $\mathrm{NTL}_{c}$ and (f) $\mathrm{NTL}_{i}$ datasets. 


\begin{tabular}{|l|c|c|c|}
\hline Dataset & BPSO & BFA & BHS \\
\hline \hline GLI-85 & 0.3130 & 0.5629 & 0.1425 \\
SMK-CAN-187 & 0.1829 & 0.2012 & 0.4432 \\
TOX-171 & 0.1742 & 0.1500 & $\mathbf{0 . 0 1 1 2}$ \\
AR10P & $\mathbf{0 . 0 0 2 3}$ & $\mathbf{0 . 0 1 9 7}$ & 0.0573 \\
NTL $_{c}$ & 0.3281 & 0.4769 & 1.2290 \\
NTL $_{i}$ & 0.1957 & 0.3318 & 1.2290 \\
\hline
\end{tabular}

Table 3 Wilcoxon Signed-Rank Test evaluation: p-values computed between BFPA, BPSO, BFA and BHS.

From Figure 3, it is possible to observe BPSO has been the technique with the fastest convergence rate, followed by BFA and BFPA. However, the good BPSO performance over the feature selection process does not seem to enhance a lot its final accuracy over the test set, as displayed in Figure 2. In addition, BHS has the slowest convergence process, since it updates only one agent (harmony) per iteration, which turns it fast considering the execution time, but it tends to be slower for convergence. Figure 4 displays the execution time for all considered optimization techniques. Though it is possible to observe BFPA has been one of the slowest techniques, since it is the only one that employs Lévy flights to move pollens across the search space, which can increase the computational burden slightly. It is also observed that BFPA in general produces better results in terms of accuracy, as seen in Figure 2.

In addition, Figure 5 displays the mean number of selected features for each dataset. It is possible to observe BHS has selected the fewest number of features, followed by BPSO. However, as we have high dimensional datasets (in case of GLI85, SMK-CAN-187, TOX-171 and AR10P), the absolute number do not differ a lot from all techniques. It seems all techniques have been similar to each other considering the recognition rate, except for the computational load and convergence speed.

\section{Conclusions}

In this work, we considered the problem of feature selection as being an evolutionarybased optimization task constrained on a boolean lattice. The idea is to represent each possible solution as a string of bits, in which each of them denotes whether a feature will be used no compose the final set or not.

We have evaluated a recent approach called Flower Pollination Algorithm to this task in six datasets, being compared against with the well-known Particle Swarm Optimization, Harmony Search and Firefly Algorithm. The experimental setting has evaluated the recognition rates, convergence speed, number of selected features and computational load. All techniques have obtained similar recognition rates, being PSO the one with the fastest convergence process, and HS the one with lowest com- 


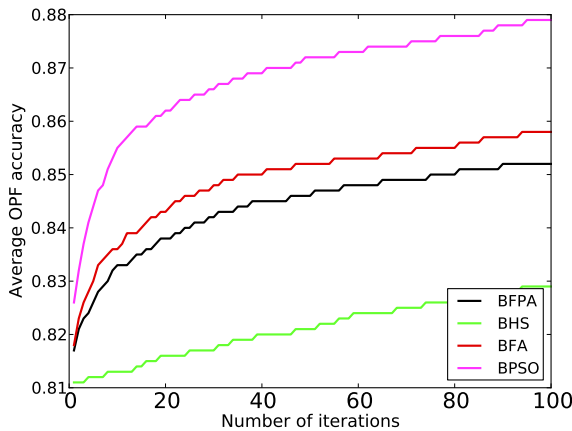

(a)

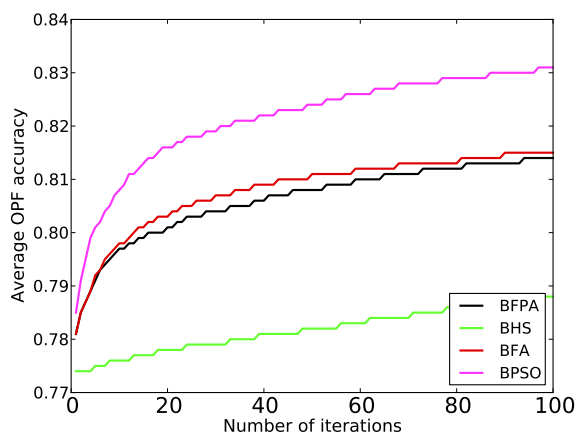

(c)

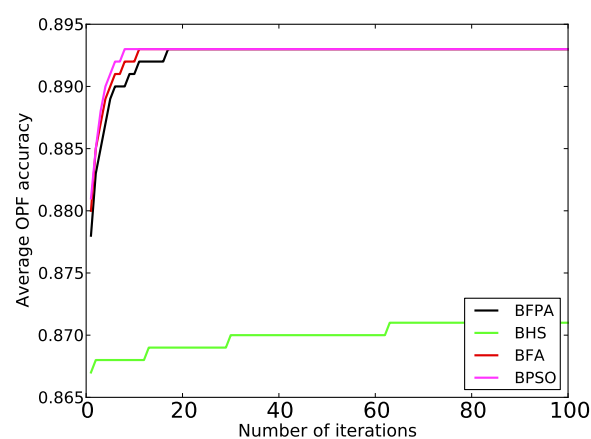

(e)

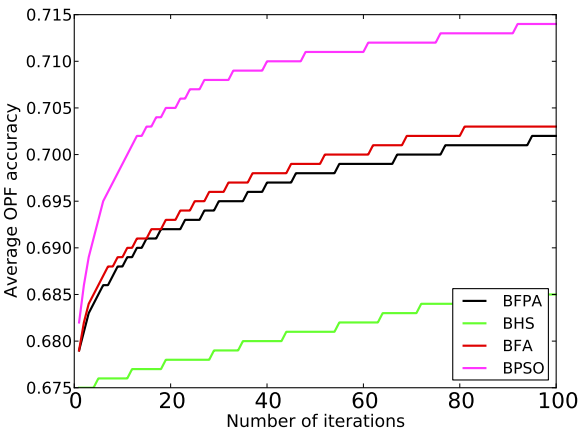

(b)

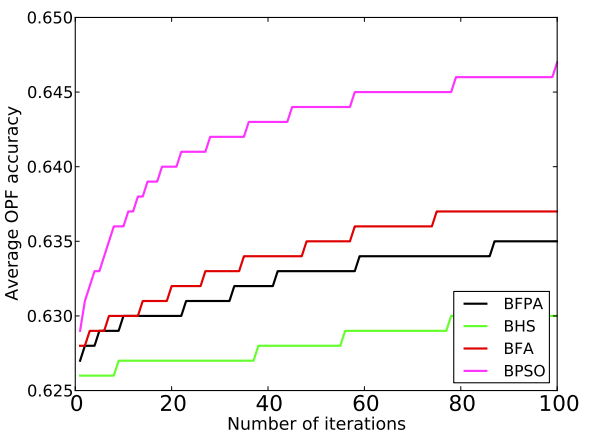

(d)

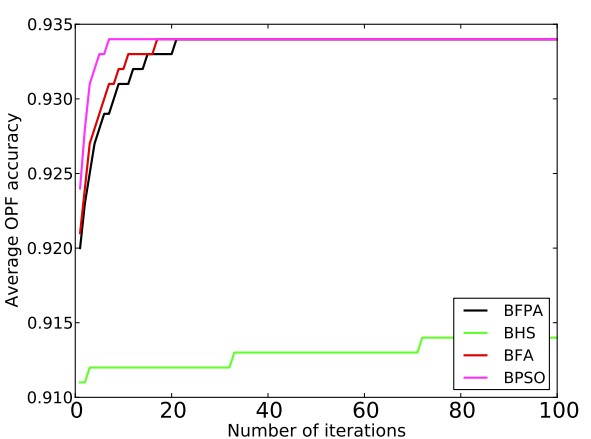

(f)

Fig. 3 Average convergence rate considering the OPF accuracy over the feature selection step for (a) GLI-85, (b) SMK-CAN-187, (c) TOX-171, (d) AR10P, (e) NTL $\mathrm{N}_{c}$ and (f) $\mathrm{NTL}_{i}$ datasets.

putational cost. Therefore, we have showed FPA is also suitable for feature selection tasks, since its results are comparable to the ones obtained by some state-of-the-art evolutionary techniques. Future works will be guided to observe the behavior of FPA regarding its parameters, as well as hybrid versions of it. 


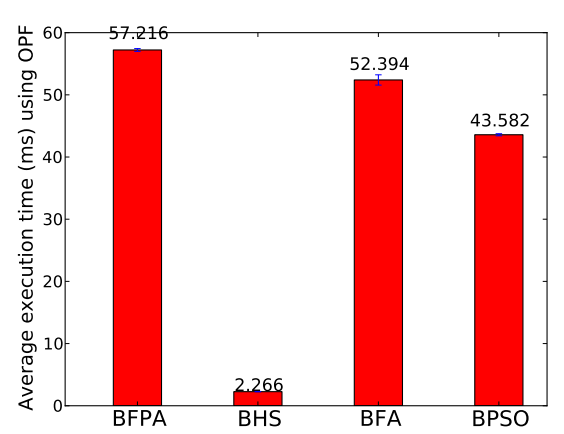

(a)

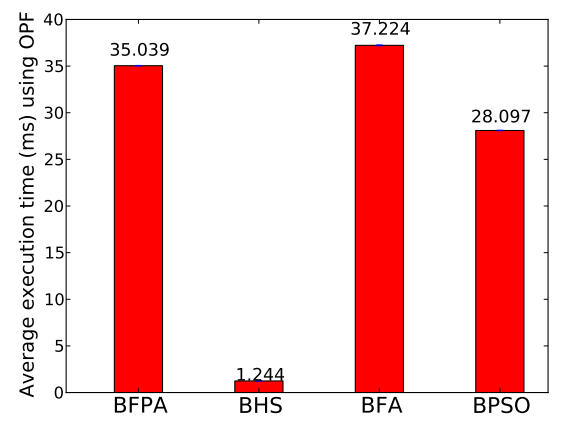

(c)

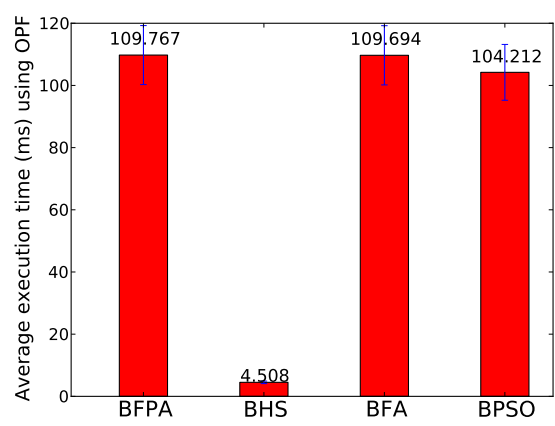

(e)

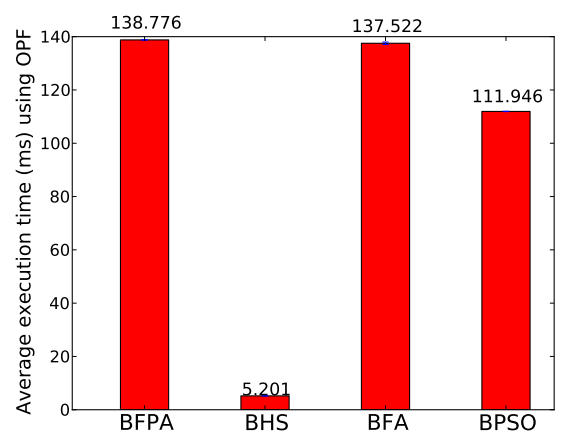

(b)

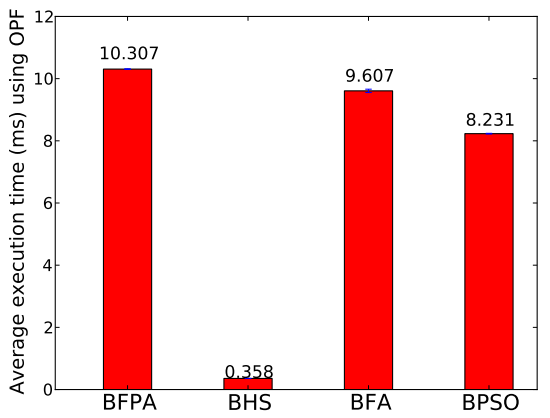

(d)

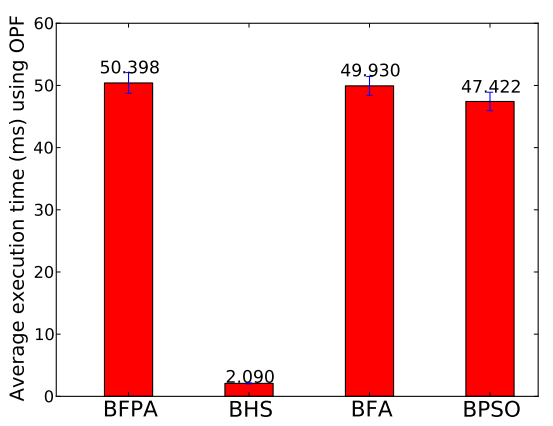

(f)

Fig. 4 Average execution time (ms) for feature selection considering (a) GLI-85, (b) SMK-CAN187, (c) TOX-171, (d) AR10P, (e) $\mathrm{NTL}_{c}$ and (f) $\mathrm{NTL}_{i}$ datasets.

\section{References}

[1] Bababdani BM, Mousavi M (2013) Gravitational search algorithm: A new feature selection method for $\{\mathrm{QSAR}\}$ study of anticancer potency of imidazo[4,5- 


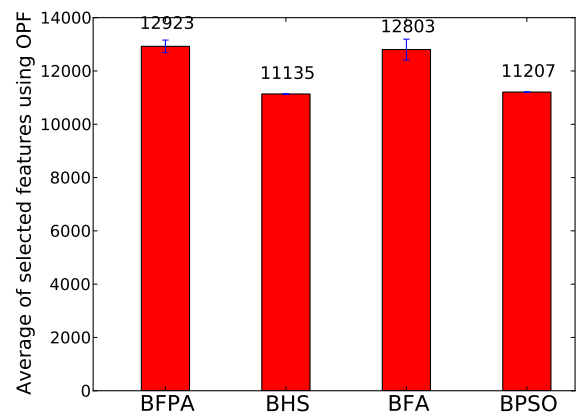

(a)

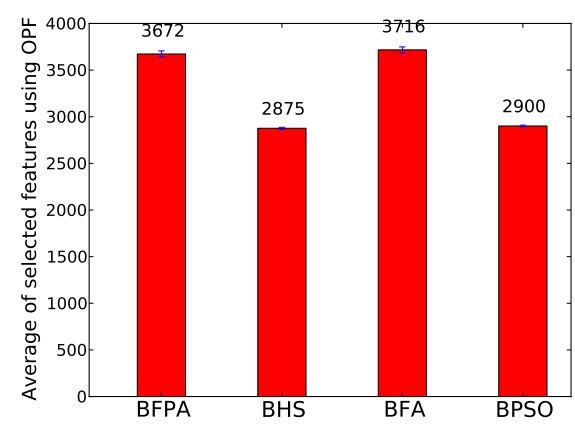

(c)

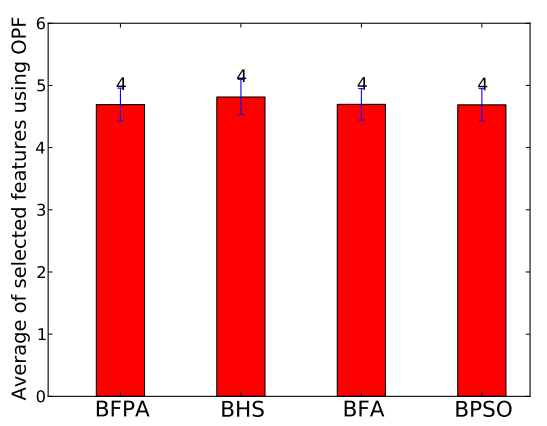

(e)

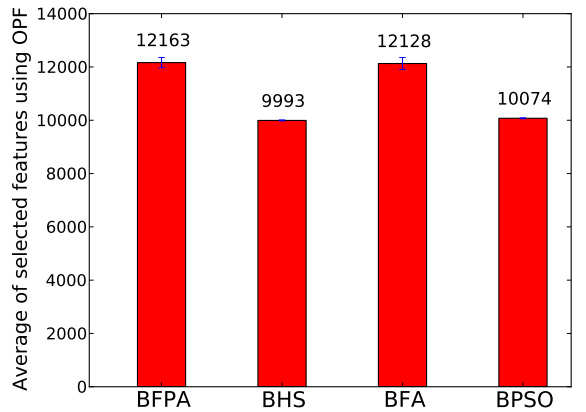

(b)

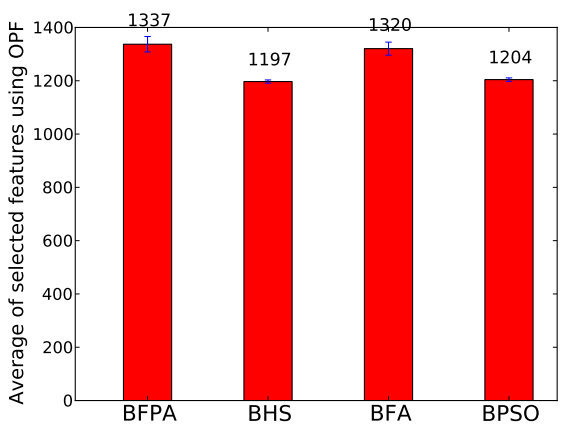

(d)

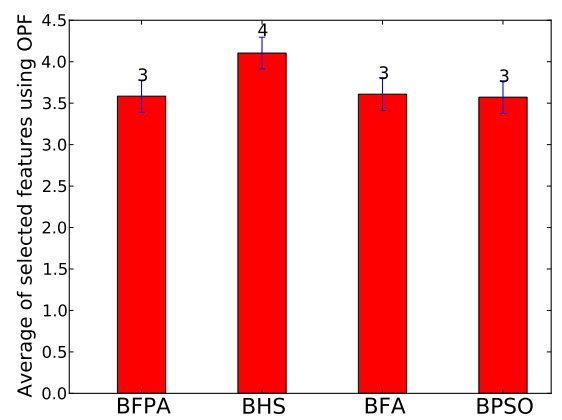

(f)

Fig. 5 Mean number of selected features considering (a) GLI-85, (b) SMK-CAN-187, (c) TOX171, (d) AR10P, (e) $\mathrm{NTL}_{c}$ and (f) $\mathrm{NTL}_{i}$ datasets. The numbers have been truncated for sake of presentation.

b]pyridine derivatives. Chemometrics and Intelligent Laboratory Systems 122(15):1-11 
[2] Chen B, Chen L, Chen Y (2013) Efficient ant colony optimization for image feature selection. Signal Processing 93(6):1566-1576, special issue on Machine Learning in Intelligent Image Processing

[3] Duda RO, Hart PE, Stork DG (2001) Pattern Classification, 2nd edn. Wiley \& Sons, Inc., New York, NY, USA

[4] Falcon R, Almeida M, Nayak A (2011) Fault identification with binary adaptive fireflies in parallel and distributed systems. In: Evolutionary Computation (CEC), 2011 IEEE Congress on, pp 1359-1366

[5] Firpi HA, Goodman E (2004) Swarmed feature selection. In: Proceedings of the 33rd Applied Imagery Pattern Recognition Workshop, IEEE Computer Society, Washington, DC, USA, pp 112-118

[6] Geem ZW (2009) Music-Inspired Harmony Search Algorithm: Theory and Applications, 1st edn. Springer Publishing Company, Incorporated

[7] Kabir M, Shahjahan M, Murase K (2009) An efficient feature selection using ant colony optimization algorithm. In: Leung C, Lee M, Chan J (eds) Neural Information Processing, Lecture Notes in Computer Science, vol 5864, Springer Berlin Heidelberg, pp 242-252

[8] Kennedy J, Eberhart RC (2001) Swarm Intelligence. M. Kaufman

[9] Marinakis Y, Marinaki M, Matsatsinis N (2009) A hybrid discrete artificial bee colony - GRASP algorithm for clustering. In: Proceedings of the International Conference on Computers Industrial Engineering, pp 548-553

[10] Oh IS, Lee JS, Moon BR (2004) Hybrid genetic algorithms for feature selection. IEEE Transactions on Pattern Analysis and Machine Intelligence 26(11):1424-1437

[11] Palit S, Sinha SN, Molla MA, Khanra A, Kule M (2011) A cryptanalytic attack on the knapsack cryptosystem using binary firefly algorithm. In: Computer and Communication Technology (ICCCT), 2011 2nd International Conference on, pp 428-432

[12] Papa JP, Falcão AX, Suzuki CTN (2009) Supervised pattern classification based on optimum-path forest. Intl Journal of Imaging Systems and Tech 19(2):120-131

[13] Papa JP, Falcão AX, Albuquerque VHC, Tavares JMRS (2012) Efficient supervised optimum-path forest classification for large datasets. Pattern Recognition 45(1):512-520

[14] Ramos CCO, Souza AN, Chiachia G, Falcão AX, Papa JP (2011) A novel algorithm for feature selection using harmony search and its application for nontechnical losses detection. Computers \& Electrical Engineering 37(6):886-894

[15] Ramos CCO, De Souza A, Falcão A, Papa J (2012) New insights on nontechnical losses characterization through evolutionary-based feature selection. Power Delivery, IEEE Transactions on 27(1):140-146

[16] Ramos CCO, de Souza AN, Falcão AX, Papa JP (2012) New insights on nontechnical losses characterization through evolutionary-based feature selection. IEEE Transactions on Power Delivery 27(1):140-146

[17] Rodrigues D, Pereira LAM, Almeida TNS, Papa JP, Souza AN, Ramos CCO, Yang XS (2013) A binary cuckoo search algorithm for feature selection. In: 
Proceedings of IEEE International Symposium on Circuits and Systems, pp 465-468

[18] Rodrigues D, Pereira LAM, Nakamura RYM, Costa KAP, Yang XS, Souza AN, Papa JP (2013) A wrapper approach for feature selection based on bat algorithm and optimum-path forest. Expert Systems with Applications 41(5):2250-2258

[19] Rodrigues D, Pereira LAM, Papa JP, Ramos CCO, Souza AN, Papa LP (2013) Optimizing feature selection through binary charged system search. In: Proceedings of 15th International Conference on Computer Analysis of Images and Patterns, pp 377-384

[20] Schiezaro M, Pedrini H (2013) Data feature selection based on artificial bee colony algorithm. EURASIP Journal on Image and Video Processing 2013(1):1-8

[21] Sivagaminathan RK, Ramakrishnan S (2007) A hybrid approach for feature subset selection using neural networks and ant colony optimization. Expert Systems with Applications 33(1):49-60

[22] Wilcoxon F (1945) Individual comparisons by ranking methods. Biometrics Bulletin 1(6):80-83

[23] Yang J, Honavar V (1998) Feature subset selection using a genetic algorithm. IEEE Intelligent Systems and their Applications 13(2):44-49

[24] Yang XS (2010) Firefly algorithm, stochastic test functions and design optimisation. International Journal Bio-Inspired Computing 2(2):78-84

[25] Yang XS (2012) Flower pollination algorithm for global optimization. In: Proceedings of the 11th International Conference on Unconventional Computation and Natural Computation, Springer-Verlag, Berlin, Heidelberg, pp 240249

[26] Yang XS, Karamanoglu M, He X (2014) Flower pollination algorithm: A novel approach for multiobjective optimization. Engineering Optimization 46(9):1222-1237 